\title{
Models and Mechanisms of Aortic Development and Disease
}

\author{
RL Goodwin ${ }^{1}$, AN Evans ${ }^{1}$, L Junor ${ }^{1}$, SV Biechler ${ }^{2}$, MJ Yost ${ }^{3}$, Varun Krishnamurthy ${ }^{4}$, Robert B. \\ Hinton $^{5}$, Kelsey Maddy ${ }^{6}$, and JD Potts ${ }^{1}$ \\ 1. Deaprtment of Cell Biology and Anatomy, School of Medicine University of South Carolina, \\ Columbia, SC. USA \\ 2. Bose, Electroforce Systems Group. Eden Prairie, MN. USA \\ ${ }^{3 .}$ Department of Surgery, Medical University of South Carolina. Charleston, SC. USA \\ ${ }^{4}$ Department of Bioengineering, Rice University. Houstin, TX. USA \\ ${ }^{5}$ Department of Pediatrics, University of Cincinnati. Cincinnati, OH. USA \\ 6. Department of Biomedical Engineering, University of Arizona. Phoenix, AZ. USA
}

Cardiovascular malformations are the most common and deadly of birth defects in humans and unfortunately very little are known of their genesis and few treatment options are currently available. Furthermore, numerous "Adult" cardiovascular diseases (CVDs) have their origins in embryonic development. For some CVDs this is developmental origin is straightforward as is the case with bicuspid aortic valves. However, in other CVDs such as aortic aneurisms, the role of dysmorphogenesis is not well appreciated. Thus, determining the cellular and molecular mechanisms of cardiovascular development will enable novel pharmacological and cellular therapies to treat these devastating and debilitating disorders.

Development of the heart and great arteries is a complex process that involves a number different progenitor cell types. A simple heart tube is formed as the left and right anterior lateral splanchnic mesoderms (primary heart fields) fold ventrally and form the primary heart tube. The primary heart tube begins spontaneous contraction as soon as it is formed, functioning to perfuse the growing embryo. Without this hemodynamic function, the embryo will cease development. As cardiac development continues, cells from the second heart field contribute to primary heart tube at both the venous and arterial poles. Cells from the proepicardium grow onto the exterior of the heart forming the epicardium. We and others have shown that a subset of these epicardial cells dive into the myocardium and differentiate into coronary vascular smooth muscle cells and endothelial cells. During the formation of the pulmonary artery and aorta, neural crest cells (NCC) migrate into the developing septum of the truncus arteriosus, playing a vital role in the formation of the arterial pole of the heart. Thus, the adult heart is an amalgamation of cell types. As the identity of these various progenitors is only now becoming resolved, determining the specific roles that these cells play during heart development is the next phase of discovery. Investigation of the development of the cardiovascular system offers another challenges in addition to identification of the cell types that contribute to the process, namely a mechanically dynamic environment created by the contraction of the heart and the resulting blood flow.

To meet these challenges our group has used a number of microscopic techniques and combination of in vivo and in vitro approaches. Using a mouse model of aortic aneurism, we have carried out 3D reconstructions of wildtype and diseased mouse aortas using markers for NCC-derived cells, smooth muscle cells, elastin. These studies revealed not only the 3D geometry of the aortic root, but also the fragmented arrangement of NCC-derived cells and smooth muscle cells, which spatially correlated with elastin fragmentation observed in the aortic aneurisms (Figure 1). 
In related studies we have created an in vitro model of aortic development using a tubular collagen scaffold in which tissues can be cultured under a variety of flow conditions. In these investigations, we cultured the truncus arteriosus from chick embryos under different flow conditions (normal vs pathological) to determine the role of fluid flow on outflow tract morphogenesis. Analysis of these experiments found that expression and localization of important signalling and extracellular matrix proteins was perturbed in the cases of pathological flow. Additionally, the morphology of the explant was altered under pathological flow conditions (Figure 2).

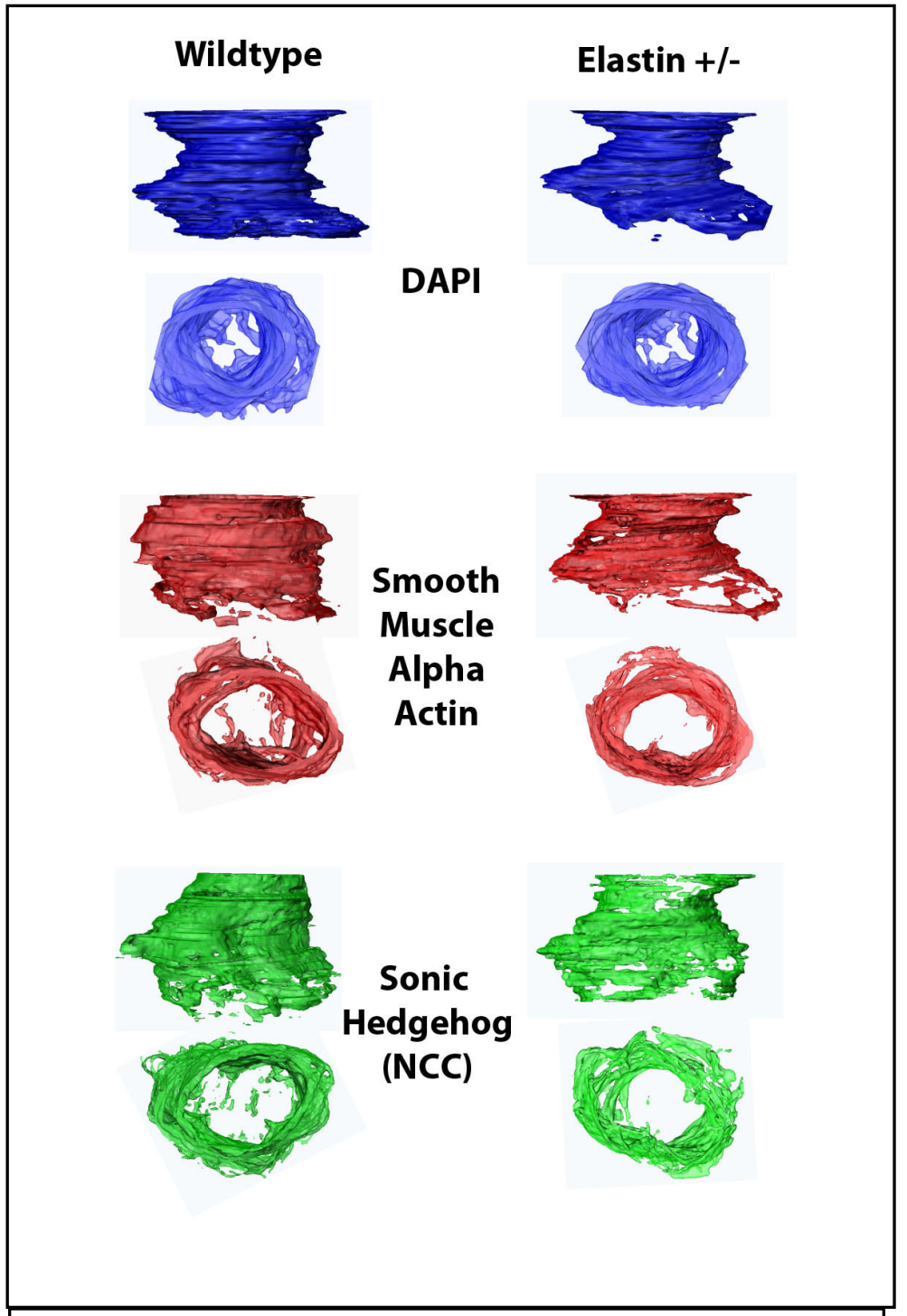

Figure 1. 3D Reconstructions of mouse aortas. Wildtype and elastin heterozygous (+/-) aortas were sectioned and stained with DAPI, smooth muscle alpha actin, and sonic hedgehog. Reconstructions revealed fragmented arrangements of NCC-derived cells and smooth muscle cells.

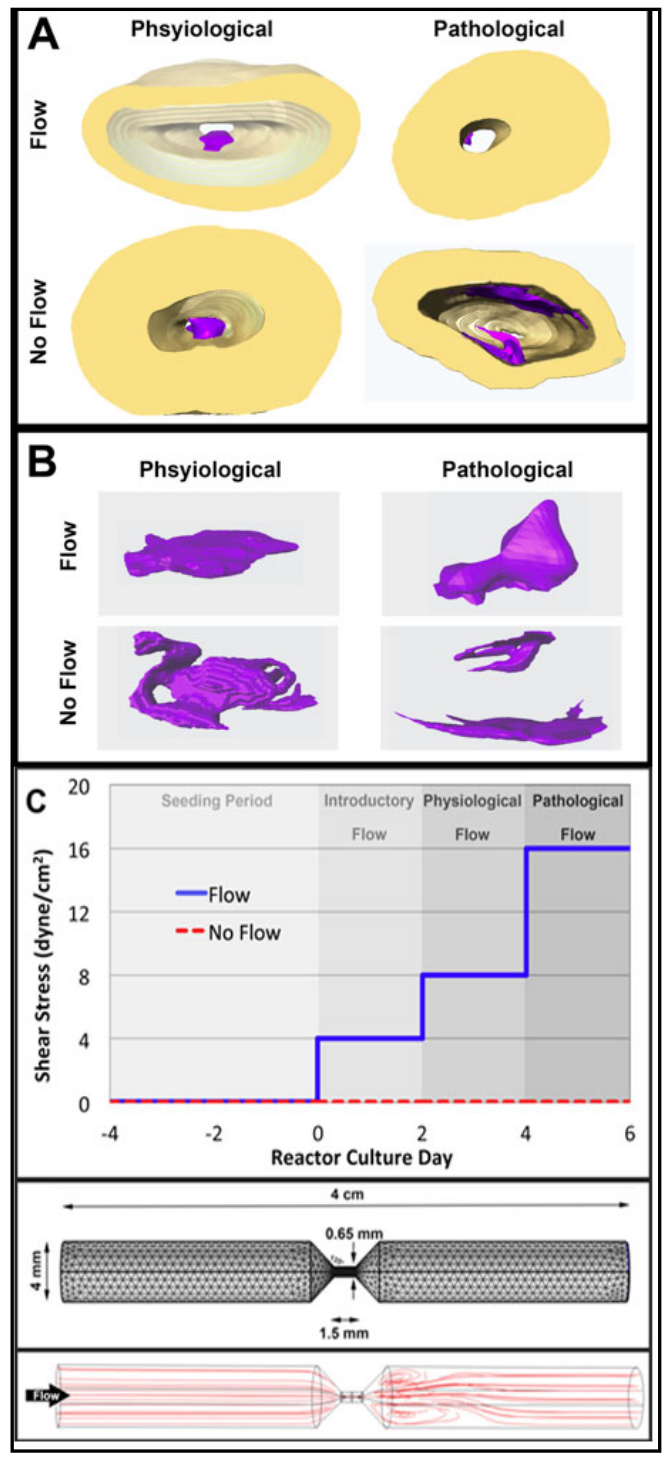

Figure 2. 3D in vitro system to study outflow tract development in the presence of fluid flow. 